\title{
Leveraging Virtual Concepts during Pandemic
}

\section{Wahag Al Mashaer Osman Mahgoub ${ }^{1}$, Noha Saleh OS Ahmed ${ }^{2 *}$ and Sherman Jabonete Dumaguin ${ }^{3}$}

${ }^{1} \mathrm{PhD}$ Candidate, MBA, BS-IT Nursing Informatics Project Manager, Nursing Informatics Department Hamad Medical Corporation, Qatar

${ }^{2}$ Nursing Informatics Research Specialist, Nursing Informatics Department Hamad Medical Corporation, Qatar

${ }^{3}$ RN Nursing Informatics Education Specialist, Nursing Informatics Department Hamad Medical Corporation, Qatar

\section{Research Article \\ Volume 3 Issue 6}

Received Date: November 27, 2020

Published Date: December 08, 2020

DOI: $10.23880 /$ jqhe-16000193

*Corresponding author: Noha Saleh Othman Saleh Ahmed, RN Nursing Informatics Education Specialist, Nursing Informatics Department Hamad Medical Corporation, Qatar, Email: NAHMED10@hamad.qa

\section{Abstract}

The world is undergoing many technological innovations which progressively take place in many industries. Virtual concepts are among the innovations that are frequently handled in many studies as a promising innovation.

The existence of COVID-19 pandemic had enforced healthcare organizations to adopt virtual concepts in providing education, training and support for their clinical staff. In this context, this study aimed to assess the effectiveness of virtual tools used during pandemic on the quality of the services provided by Nursing Informatics Department - Hamad Medical Corporation Qatar.

The data analyzed in this study was collected by a questionnaire to end-users and interviews questions to clinical leaders. Sampling was created as of 17\% (541 /3190) of the end users and all the nursing leaders assigned in the treatment sites.

According to the study results, it was concluded that virtual concepts implementations were satisfying $80 \%$ of the respondents where they have agreed on its effectiveness. It was also perceived that the participants were more engaged in their learning process which is among the factors that impact the participants' high satisfaction rate.

Additionally, participants agreed that the use of the virtual tools enabled effective utilization of resources to face the challenging demands of nursing workforce.

The paper recommends conducting further studies on the factors influencing virtual team performance and motivation and its impact on learning outcomes and other studies on the impact of applying the same technology on individuals with disabilities or had different inadequacies and assesses the outcomes.

In conclusion, the new normal has set the path for transforming the training and support provided to clinicians through applying virtual concepts where it is required to forecast the necessary trainings needed in the future to streamline the digital health education using telemedicine.

Keywords: Virtual Concepts; Clinical Training; Remote Support; Virtual Teams; Quality of Service 


\section{Introduction}

Virtual concept refers to the reliance by actors on ICT tools to mimic the real world and operate independent of time and space to attain common goals [1].

A virtual team can formally be defined as a group of geographically and/or organizationally dispersed coworkers assembled using a combination of information and communications technologies for accomplishing an organizational task [2-4].

The traditional method of in-person training or meeting becomes undependable for a time in circumstances where physical interaction is admissible. Virtual and mobile tools are replacing the conventional approach and enabling the employees to easily interact in business environment without physical restrictions $[5,6]$.

The existence of COVID-19 crisis has conveyed a new reality where it catalyzed the development of virtual interaction. This pandemic introduced new and unique challenges as it continues to spread rapidly with an emerging need to introduce new approach in providing services [7-9].

To train the staff and keep in touch with other stakeholders with the majority are under a stay-at-home order, virtual meetings, online support and e-Learning have grown exponentially.

Today, the advantages of online training are more important than ever. It will make the opportunities available for personal and professional development nearly endless $[10,11]$.

This study will describe the alternative tools that can be used to run the business during pandemic situation providing a new approach for training collaboration and getting in touch with people remotely. This paper will also discuss the impact of these tools on the quality and effectiveness of the services provided to nursing staff in Hamad Medical Corporation (HMC).

\section{Study Description}

The onset of the year 2020, the world has faced COVID-19 pandemic. The health crisis has impacted different nations. Multiple organizations, national and international, private and government sectors, health organizations and individuals have been working together to fight against this pandemic.

The State of Qatar is no exemption to the worldwide outbreak brought about by COVID-19. Hamad Medical
Corporation (HMC), the largest healthcare provider, has devoted several facilities and services to treat and manage numerous COVID-19 patients. The essential opening of treatment sites has expanded the health system capacity and resources to accommodate the rapid influx of sick individuals. The corporation also started to assemble and distribute nursing staff classified as regular new hires, volunteers, HMC nurses and non-HMC nursing staff from all over the state.

Nursing Informatics Department (NI), on the other hand, is also engaged in battling the crisis through allocation of its resources to different treatment sites, conduction of customized Clinical Information System (CIS) training programs suitable to nursing staff on board, involvement in CIS data collection, building, testing, validation and implementation in new treatment sites.

The progression of COVID19 crisis has required a substantial change of operational tasks and manpower of NI Department to support the plans and strategies of HMC.

The department has also established the NI COVID-19 Response Team to develop contingency plans and revolutionize its approach to enhance the quality of services. It utilized virtual concepts to avoid disruption of its vital operations during the involvement in the battle against COVID-19.

The main objectives of the department were:

- Create a foundation of online training programs for nursing staff to function confidently and efficiently.

- Provide the correct privileges for nursing staff in accessing the electronic health record after training conduction.

- Provide full support to nursing staff through a comprehensive support model delivering services 24/7.

- Collaborate with various departments and specialties to ensure a smoother flow of its services.

- Training nursing staff through online platform using the available technology in HMC was the first to start up with.

Developing virtual training programs was the next phase where the Operational and Training Teams adapted to better utilize the virtual teaching environment customizing training materials according to the end-user's requirements. The virtual sessions started in April 2020, just after the pandemic began to prevent necessary physical interaction.

Trainers are leveraged to conduct simultaneous, practical training sessions through virtual platforms achieving 91\% effectiveness level. With the leadership team managing the entire process, they ensured alignment and consistency of delivery for each class to end-users according to their needs. 
Additionally, and as another e-learning innovation, Nursing Informatics provided all the necessary materials that assisted nursing staff throughout the patient journey using the Clinical Information System (CIS) through NI CIS e-Library. It is a user-friendly reference online tool that delivered a collection of CIS educational materials, training modules and news updates designed to support every array of nursing staff across HMC. The interface and features made learning simple and easy. It offered a wide range of educational materials from various nursing specialties that were customized according to their nursing workflows.

As a complementary step, the nurses were provided online courses on basic concepts of computer and on how to use the online tools to assist them in dealing with the Clinical Information System while delivering care.

NI staffs have been passionately delivering on-theground support to HMC and non-HMC treatment sites 24/7 for nursing staff. In other facilities, they have replaced physical support by online support, assigning a roundthe-clock on call service and dedicated team known as the COVID-19 NI Hotline Team to respond to all nursing staff's technical, system and workflow queries and provided them with the utmost assistance to deliver a high quality of care to the patients using Virtual Network Computing (VNC), a desktop-sharing system to remotely control another computer.

NI management and leads have constantly worked with the corporate departments on the aspect of training to the much-needed nursing workforce. The department also was actively engaged to deliver the needed requirements of other departments through dashboard development, generating and analyzing nursing related data and provided reports to facilitate planning and decision making for safe delivery of healthcare services in HMC.

\section{Aim}

The study aims to assess the effectiveness of virtual tools used during pandemic on the quality of the services provided.

\section{Objectives}

- To efficiently utilize time and resources while delivering simultaneous tasks.

- To evaluate staff satisfaction on the approach used.

- To assess the effectiveness of online training and support.

\section{Data Collection}

A questionnaire for end-users as well as 5 interview questions for nursing leaders is distributed electronically to measure the effectiveness of virtual tools used and its impact on the quality of services provided to nursing staff across Hamad Medical Corporation, Qatar. The questions in the survey are measured using 5-point Likert scale where 1 denoted "Strongly Disagree" and 5 "Strongly Agree" including demographic data and suggestions.

\section{Sample}

The study targeted $17 \%$ of the end users out of the entire population (541 / 3190) who were assigned in treatment sites. Similarly, there are 18 out of 27 nursing and clinical leaders have responded to the interview questions.

\section{Results and Analysis}

Nursing Informatics Department is constantly evaluating its services and monitoring its Key Performance Indicators (KPIs) that demonstrates the effectiveness of the overall performance of department's activities. The results in the two surveys exhibited the findings from heterogeneous group of participants for a response timeframe of two weeks.

\section{Nursing and Clinical Leaders Survey}

The aim of this survey is to measure the effectiveness of the quality of services and support provided during the COVID-19 pandemic state. The structured questionnaire is categorized into 4 areas.

Support: All respondents have perceived the proactive participation and contribution of NI team in their facility. They have expressed the efficient involvement in creating new processes, procedures and workflow, sufficing technical requirements and training including physical and online support provided to their nursing staff. One of the highlighted responses is "They provided excellent support in a timely manner, anticipated our needs and delivered on their promises on every occasion. They provided training over weekends allowing staff to be deployed in a timely manner to meet the service needs".

Communication: Most of the participants have conveyed the prompt response of NI team to queries and concerns of nursing staffs through on call, NI COVID-19 Hotline and online access to the nurses' stations through VNC. One of the highlighted responses is "Communicating thru email or hotline number, whenever address any concern or issues related to Nursing informatics got prompt response with suggestion or solution".

Satisfaction: All respondents expressed their overall satisfaction with the quality of service delivered to nursing staffs. They responded effectively to resolve CIS issues and other Nursing Informatics related concerns, provided staff training and involved in CIS implementation in the facility that 
enhanced the quality of patient care. One of the highlighted responses is "Our department has received excellent service and help from the NI operational team. They have been always ready to help, explain and guide us, with is especially important at this difficult time"

Identifying Gaps: All participants conveyed that there were no identified gaps pertaining to the services provided by Nursing Informatics. However, the respondents are cooperative to discuss if there are any known gaps in the future.

\section{Nursing Staff Survey}

Nursing Informatics has conducted the survey to improve and to measure the effectiveness of its services on the aspect of training, educational resources provided, training benefits and support.

More than $80 \%(433 / 541)$ of the respondents have expressed the overall effectiveness of CIS trainings based on the trainer's knowledge, clearly defined objectives, relevancy and accessibility of online training. $77 \%$ (417/ 541) of the respondents stated that the time conducted for trainings were enough whereas $9 \%$ specified that the time allotted was not enough and it requires longer hours. The urgency of nursing workforce in treatment facilities required lesser time for orientation and trainings before their deployment. Thus, the CIS training for new hires was modified to cope with these requirements. On the other hand, the question was skipped by $15 \%$ of the participants (Figures $1 \mathrm{a}-1 \mathrm{~h}$ ). a

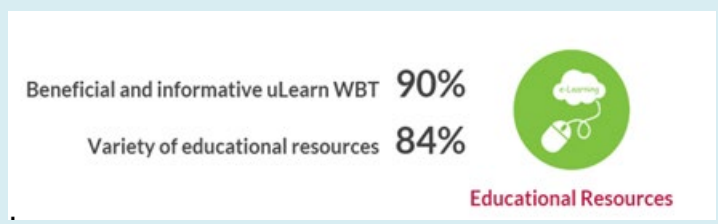

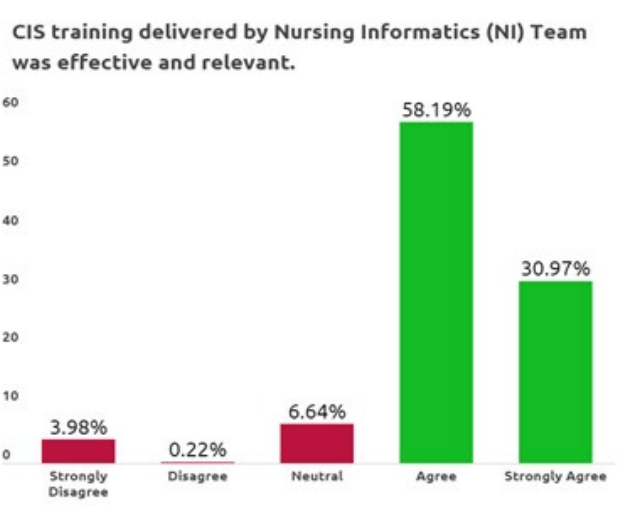

b

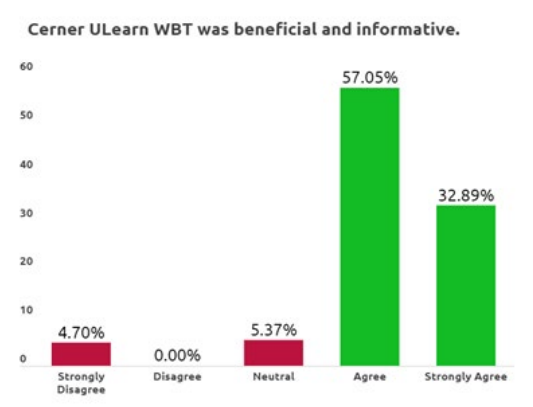




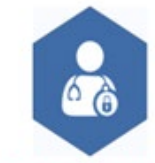

Training Benefits

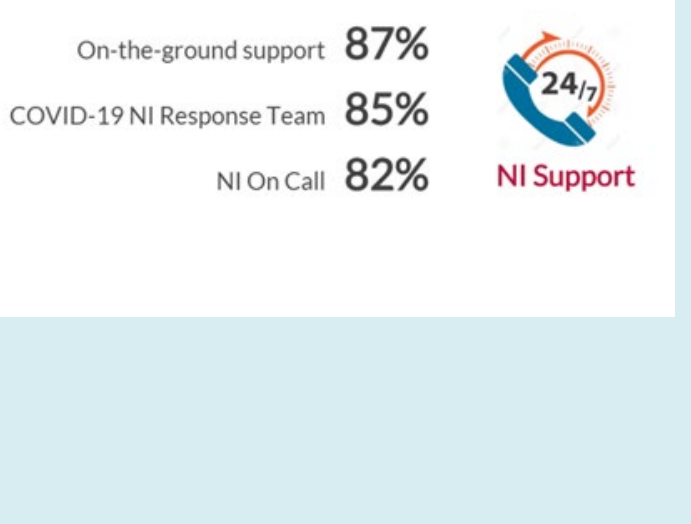

g
I was able to ensure patient safety through proper

documentation in the system.

60

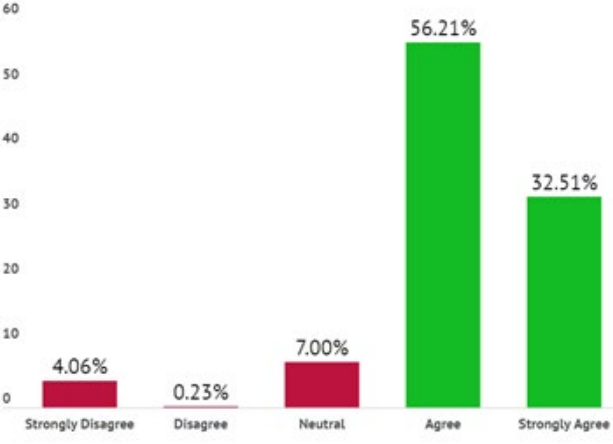

$\mathrm{f}$

COVID-19 NI Response Team (NI call center) is helpful to resolve $\mathrm{CIS}$ and $\mathrm{NI}$-related concerns.

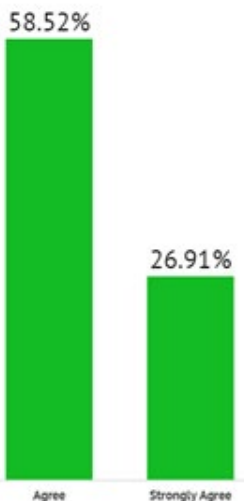

$\mathrm{h}$

Figures 1a-h: Percentages of patient's electronic health records.

Apart from 90\% (487/ 541) of uLearn Web Based Training (WBT) being beneficial and informative, the variety of educational resources offered for training and support was $84 \%$ (454/541) It was realized that these resources were valuable and helpful to most of the respondents during their CIS training.

Upon recognizing the effectiveness of the trainings, an exceeding $80 \%$ (433/541) of the participants have benefited by way of having confidence in documentation, ensuring patient's safety and observing privacy and confidentiality of patient's electronic health records.

Most of the respondents have agreed that they have been supported by Nursing Informatics staff in their facilities whether on-the-ground or online. Over $80 \%$ (433/541) of the nursing staff affirmed that they have received great support and guidance.

\section{Conclusion}

The use and applying virtual concepts have grown exponentially in the past decade. Advancement in technology has made the virtual tools more accessible and highly utilized. Virtual concepts should be adopted as a proactive measure to improve staff competence and should not be an emergency approach referred to during critical situations.

Despite the challenges posed by the COVID-19 pandemic, utilizing virtual concepts provided great promise to the 
Nursing Informatics Department to perform tasks remotely and support clinicians to provide high quality of care.

This study has highlighted essential implications for the current health crisis produced by COVID-19 pandemic.

The satisfaction among nursing leaders for the services provided was very high in addition to their recognition for the real-time communication and the online support provided during the pandemic.

While the study did not reveal significant differences in actual training outcomes, majority of the respondents have agreed on the effectiveness of online trainings and that they gained the required knowledge. The staff acquired confidence in documentation which ensure patient's safety, privacy and confidentiality. Further perception, the participants were more engaged in their learning and assess the overall process as beneficial and significant.

Despite the high satisfaction rate of the participants towards the training provided, yet a few participants emphasized the need to increase the time allotted for training.

Additionally, the application of virtual concepts assisted in the effective utilization of resources with the high demand to allocate more resources for training and support.

The utilization of virtual concepts in the current pandemic situation lies in the convenience and benefits it offers to the patient and healthcare providers. In order to provide safety for healthcare providers, they must be well trained on how to use this technology. Nursing Informatics should forecast the future training needs to streamline the digital education and construct the educational modules with technologies to enhance learning experience.

\section{Recommendation}

The COVID-19 pandemic presents an unprecedented challenge in providing services, training and support for healthcare providers as a result of the high demand and the limited workforce available to adjust to the uncertainty of the situation. However, amidst the uncertainty, utilizing virtual concepts provided unique opportunities for delivering the support and necessary training for frontline staff, enabling them to gain knowledge and skills required for delivering high quality of care. There is no doubt that the approaches taken will become the future of nursing informatics education, training and support as a supplement to traditional ways.

The study recommends developing a training model that adopts the blended learning strategy, designing interactive educational materials and engaging the stakeholders in planning and implementing the learning process to yield better learning outcomes.

However, managing such a model requires high-level of coordination which requires establishing well-planned monitoring mechanisms to detect any emerging problem to resolve it. It is also recommended to conduct pre and post -training assessment to ensure the quality of learning outcomes.

Application of virtual concept provides many benefits, but also creates various challenges, particularly associated with communication and technology. To adopt this transformation, the department should have a supportive and reliable IT infrastructure to facilitate more complex and interactive communication technologies that are becoming more popular.

Furthermore, this case study emphasizes the need for future researches studying factors influencing virtual team performance and motivation and its impact on learning outcomes. Other researches can also be conducted on the impact of applying the same technology on individuals with disabilities or had different inadequacies and assess the outcomes.

Although future researches are required to determine the effectiveness and applicability of virtual tools in conducting all types of nursing education, yet this study demonstrated that virtual concepts can open new horizon for transformation of education, training and support for clinical staff and helps them providing a high quality of care.

\section{References}

1. (2020) What is Virtual Concept. Global Publishers of Timely Knowledge.

2. Lisa Handke, Costa PL, Klonek FE, O'Neill TA, Parker SK (2020) Team perceived virtuality: an emergent state perspective. European Journal of Work and Organizational Psychology.

3. Morrison Smith, Sarah Ruiz, Jaime (2020) Challenges and barriers in virtual teams: a literature review. SN Applied Sciences 2.

4. Meluso J, Johnson S, Bagrow JP (2020) Making Virtual Teams Work: Redesigning Virtual Collaboration for the Future. pp: 1-14.

5. Delloite Report (2020) Remote Collaboration Facing the challenges of COVID-19. pp: 1-13. 
6. Amy C, Nick Y, Gerard G (2016) The digital workforce and the workplace of the future. Academy of Management Journal 59: (3) 731-739.

7. Dulebohn JH, Hoch JE (2017) Virtual teams in organizations. Human Resource Management Review. 27(4): 569-574.

8. Holtz K, Orengo Castella V, Zornoza Abad A, González Anta B (2020) Virtual team functioning: Modeling the affective and cognitive effects of an emotional management intervention. Group Dynamics: Theory, Research, and Practice 24(3): 153-167.
9. Han SJ, Chae C, Macko P, Park W, Beyerlein M (2017) How virtual team leaders cope with creativity challenges. Eur J Train Dev.

10. Algesheimer R, Dholakia UM, Gurău C (2011) Virtual team performance in a highly competitive environment. Group \& Organization Management 36: 161-190.

11. Eisenberg J, Post C, DiTomaso N (2019) Team dispersion and performance: the role of team communication and transformational leadership. Small Group Res 50(3): 348-380. 